\title{
Práctica de ejercicio físico y su relación con el consumo de tabaco en adolescentes. Diferencias entre géneros
}

\section{Relationship between tobacco consumption and physical exercise in adolescents. Differences between genders}

\author{
Pedro Luis Rodríguez García; Francisco José López \\ Villalba; Pedro Ángel López Miñarro; Eliseo García \\ CANTÓ
}

| Facultad de Educación. Universidad de Murcia

Enviar correspondencia a:

Francisco José López Villalba

C/ José Marín Camacho, 28.

30530 CIEZA-MURCIA

paco.lopez@um.es

\section{Resumen}

El objetivo del estudio fue analizar la relación entre la práctica de actividad físico-deportiva y los hábitos de consumo de tabaco en adolescentes escolarizados, en función del género. Un total de 845 adolescentes (412 varones y 433 mujeres) representativos de la población adolescente escolarizada de la Región de Murcia, con edades comprendidas entre 14 y 17 años, participaron en el estudio. A todos ellos se les administró el International Physical Activity Questionaire para evaluar su práctica de actividad física, así como el cuestionario Youth Risk Behavior Surveillance para determinar su consumo de tabaco. Los resultados mostraron una relación significativa entre la práctica físicodeportiva y el consumo de tabaco, además de una clara diferencia en función del género. En los varones, se apreció una relación positiva y significativa entre la práctica físico-deportiva y el consumo de tabaco. Por el contrario, en las mujeres no se encontró dicha asociación. En conclusión, las diferencias en la relación entre la práctica de actividad físico-deportiva y el consumo de tabaco entre varones y mujeres están asociadas a las características de los modelos de promoción deportiva existentes en la actualidad, así como a las diferentes motivaciones de varones y mujeres para implicarse en una actividad físico-deportiva. recibido: Marzo 2012 aceptado: Octubre 2012

\section{Abstract}

The objective of this study was to analyze the relationship between physical exercise and sport activities with respect to tobacco consumption in adolescents according to gender. A total of 845 adolescents (412 males and 433 females) between 14 and 17 years of age enrolled in compulsory secondary education in Murcia (Spain) participated in the study. The International Physical Activity questionnaire was administered to all of them to determine their physical activity patterns, also the Youth Risk Behavior Surveillance questionnaire was given to evaluate their smoking behaviors. The results show a significant relationship between physical exercise and sport activity and tobacco consumption. In addition, a clear gender difference was found on tobacco use. Among males, a positive and significant relation between practice of sports and physical activity and tobacco use was found; while no association between both variables was found among females. In conclusion, differences between men and women related to practice of physical exercise and sport and tobacco consumption are associated with the characteristics of the current models used to promote practice of sports as well as the different motivations among men and women to engage in physical exercise and sport activity. 
a proliferación en los últimos años del consumo de tabaco entre los jóvenes ha despertado una gran alarma social. Las diferentes investigaciones sobre las conductas sociales de consumo confirman el aumento paulatino en la instauración y desarrollo de dicha droga legalizada, que se ha consolidado progresivamente como hábito nocivo de vida de nuestros adolescentes (Ford, Nonnemake y Wirth, 2008; Suelves, Romero y Sánchez, 2000).

El tabaquismo se perfila como una de las principales causas de morbilidad y mortalidad y, en la actualidad, se considera como un trastorno adictivo crónico (Bello, 2011; Lekuona, Salcedo, Morillas y Umaran, 2009) y recurrente registrada con el código F17 en la Clasificación Internacional de las Enfermedades (CIE-10) de la Organización Mundial de la Salud (1992). En este sentido, la Organización Mundial de la Salud (1997) reconoce que la propagación de la epidemia del tabaquismo es un problema mundial de graves consecuencias para la salud pública. El uso del tabaco es también una epidemia pediátrica, ya que los nuevos fumadores se inician principalmente durante la etapa escolar o preadolescente.

El consumo de tabaco es un hábito no saludable que conlleva una conducta de riesgo. Estas conductas de riesgo se establecen cada vez a edades más tempranas y se acentúan en las etapas iniciales de la adolescencia (Audrian-McGorven, Rodríguez y Moss, 2003; Larson, Store, Perry, NeumarkSztainer y Hannan, 2007).

La mayoría de investigaciones sociológicas realizadas en diferentes países constatan que los adolescentes contactan con el tabaco en períodos previos a la adolescencia, manteniéndose en muchos casos durante el resto de la vida (AudrianMcgovern, Rodríguez, Wileyto, Schmitz y Shields, 2006; Nistal, Prieto, Del Valle y González, 2003). La adolescencia es una etapa de la vida en la que existe una gran vulnerabilidad hacia la adquisición de hábitos nocivos para la salud, como lo es el consumo de tabaco. Diversas investigaciones constatan que la presencia de un entorno de amigos fumadores se relaciona positivamente con el hábito de consumo de tabaco (García, Carrillo, Fernández y Sánchez, 2006; Leatherdale, McDonald, Cameron y Bown, 2005; Nichols, Birnbaum, Birnel y Botvin, 2006; Robison, Dalton y Nicholson, 2006).

La adopción de un estilo de vida activo mediante una práctica regular de actividad físico-deportiva se ha asociado a la reducción de conductas de consumo de sustancias tóxicas, entre las que podemos señalar el tabaco (Bergamaschi, Morri, Resi, Zanetti y Stampi, 2002; Colmen, Barrett, Clausen, Holemn y Bejrmer, 2002).

La práctica de actividad física y deportiva está considerada como uno de los hábitos más representativos dentro de los estilos de vida saludables. Desde diversos sectores especializados se destaca el papel que un ejercicio físico adecuado posee en la prevención de la mayoría de las alteraciones producidas por nuestra sociedad marcadamente sedentaria y mecanizada. Los grandes avances en materia de investigación generados a lo largo del último siglo corroboran los efectos fisiológicos beneficiosos de una práctica de actividad física correcta, regular y sistematizada (Daniel, Cropley, Usscher y West, 2004; Pate et al., 2007; Sánchez et al., 2007; Telama et al., 2005).
Diversas investigaciones señalan que aquellos adolescentes que realizan una práctica habitual de actividad físico-deportiva se inician más tarde y tienen consumos menores de tabaco que aquellos que no practican (Aleixandre, Perello y Palmer, 2005; Halperin, Smith, Heligenstein, Brown y Fleming, 2010; lannotti, Kogan, Janssen y Boyce, 2009; Madan et al., 2005; Moore y Werch, 2005; Wilson et al., 2005). Consideramos que uno de los factores que puede incidir positivamente sobre la disminución de este hábito negativo para la salud de los adolescentes es el fomento y desarrollo de una práctica regular de actividad físico-deportiva.

En el presente estudio se ofrece un análisis de la relación establecida entre el hábito de consumo de tabaco y la práctica físico-deportiva. La originalidad de nuestra contribución se basa en la incidencia diferenciada que la práctica físico-deportiva posee en función del sexo en relación con el hábito de consumo de tabaco. Así mismo, la mayoría de investigaciones señalan diferencias significativas en la frecuencia y porcentaje de participación entre varones y mujeres. Los varones realizan más práctica deportiva que las mujeres (Gómez, Duperly, Lucemi, Gámez y Venegas, 2005; Levin, Lowry, Brown y William, 2003). Del mismo modo, encontramos diferentes motivos de práctica en función del sexo. En este sentido, los varones suelen realizar práctica deportiva bajo intereses centrados en la competición deportiva, la exaltación del ego, la aprobación social o la mejora de la condición física. Por ello, es frecuente que los contextos de práctica deportiva en varones sean de naturaleza competitiva y centrados en la práctica deportiva reglada, donde la presencia del tabaco suele ser inexistente (Moreno, Moreno y Cervelló, 2009). Por el contrario, los motivos de práctica físico-deportiva en las mujeres adolescentes difieren en gran medida de los varones, estando orientados fundamentalmente al cuidado de la estética, el control del peso corporal, la diversión y el establecimiento de relaciones afectivo-sociales (Bungum, Dowda, Weston, Trost y Pate, 2000; Castillo y Balaguer, 2004; Cechini, Méndez y Muñiz, 2003; Crisp et al., 1998; O'Loughlin, Paradis, Renaud y Sánchez, 1998; Quiles et al., 2011; Tomeo 1999).

A la luz de tales consideraciones, los objetivos de la presente investigación se centran en constatar las posibles relaciones existentes entre la práctica físico-deportiva y el hábito de consumo de tabaco en adolescentes escolarizados de edades comprendidas entre los 14 y los 17 años, así como las posibles diferencias entre varones y mujeres en la relación de ambas variables.

\section{Método}

\section{Participantes}

Mediante la aplicación de un diseño de encuestas por muestreo fue seleccionada una muestra representativa de adolescentes escolarizados de la Región de Murcia (España) entre 14 y 17 años. La muestra quedó integrada por alumnos pertenecientes a $3^{\circ}$ y $4^{\circ}$ de Educación Secundaria Obligatoria (E.S.O.) y $1^{\circ}$ de Bachillerato. Siguiendo un proceso estratifica- 
do y polietápico con diferentes unidades de muestreo (comarcas, municipios y centros escolares) se seleccionó a los sujetos mediante un procedimiento con probabilidad proporcional al tamaño, dando lugar a una muestra autoponderada, donde la selección final de los adolescentes en las aulas fue aleatoria. Fueron seleccionados 13 centros públicos de diferentes localidades de la Región de Murcia (España).

Para un nivel de confianza del 95,5\% y un error determinado de $\pm 3,2 \%$ en nuestras estimaciones, la muestra definitiva quedó formada por un total de 845 adolescentes, 51,2\% mujeres y $48,8 \%$ varones con edades comprendidas entre 14 y 17 años (tabla 1). De la totalidad de la muestra aleatoria seleccionada, fueron rechazados 28 de los sujetos $(3,24 \%)$ por no cumplimentar de forma adecuada el cuestionario autoadministrado.

Tabla 1. Distribución de la muestra en función del sexo y la edad

\begin{tabular}{|c|c|c|c|c|c|c|c|}
\hline & & & \multicolumn{4}{|c|}{ Edad } & \multirow[b]{2}{*}{ Total } \\
\hline & & & 14 & 15 & 16 & 17 & \\
\hline \multirow[t]{4}{*}{ Sexo } & Hombre & Recuento & 62 & 85 & 124 & 141 & 412 \\
\hline & & $\%$ del total & $7,3 \%$ & $10,1 \%$ & $14,7 \%$ & $16,7 \%$ & $48,8 \%$ \\
\hline & Mujer & Recuento & 71 & 94 & 139 & 129 & 433 \\
\hline & & $\%$ del total & $8,4 \%$ & $11,1 \%$ & $16,4 \%$ & $15,3 \%$ & $51,2 \%$ \\
\hline \multirow[t]{2}{*}{ Total } & & Recuento & 133 & 179 & 263 & 270 & 845 \\
\hline & & $\%$ del total & $15,7 \%$ & $21,2 \%$ & $31,1 \%$ & $32,0 \%$ & $100,0 \%$ \\
\hline
\end{tabular}

Al observar la distribución de sujetos por edades se aprecia una mayor proporción de concentración de los sujetos en las edades de 16 y 17 años, debido a que en los cursos de $3^{\circ}$ y $4^{\circ}$ de la E.S.O. se acumula un mayor porcentaje de alumnos repetidores.

\section{Variables e instrumento}

Utilizamos tres tipos de variables: sociodemográficas (sexo, edad y curso), hábitos de consumo de tabaco (ha probado alguna vez, edad de inicio, consumo habitual y cantidad de tabaco consumido) y la práctica deportiva. Utilizamos dos instrumentos de evaluación y registro para cada una de las dimensiones relacionadas en el estudio. De esta forma, para valorar la práctica de actividad físico-deportiva realizada de forma regular por los adolescentes se utilizó el formato corto del International Physical Activity Questionaire (Craig et al., 2003). Dicho cuestionario consta de 4 ítems de carácter general sobre el tiempo que dedicó a estar activo los últimos 7 días. Se realizó una adaptación cultural de términos y se llevó a cabo un proceso de traducción del inglés al español. Todo el proceso de adaptación se realizó siguiendo las recomendaciones que la International Tests Comisión (ITC) ha desarrollado para la construcción y adaptación de tests (COP-ITC, 2000; Muñiz, 1996). De acuerdo con ellas, este proceso no sólo consistió en la traducción, sino en la adecuación de los ítems formulados ajustándolos al contexto del hablante en castellano, cuidando incluso de traducir algunos términos al lenguaje coloquial propio de los adolescentes.

Las categorias de la variable resultado fueron inactivo, irregularmente activo y regularmente activo, definidas con los siguientes criterios:
1. Inactivos: personas que no habían caminado o realizado otra actividad física de intensidad moderada o vigorosa los últimos 7 días, durante al menos 10 minutos seguidos.

2. Irregularmente activos: personas que habian caminado o realizado otra actividad física de intensidad moderada o vigorosa con una duración acumulada diaria de al menos 10 minutos.

3. Regularmente activos: personas que habían caminado o realizado otra actividad física de intensidad moderada, con una duración acumulada de al menos 30 minutos al día, en esfuerzos mínimos de 10 minutos seguidos, durante 5 días o más en los últimos 7 dias, o habían realizado actividades vigorosas con una duración acumulada de al menos 20 minutos seguidos durante 3 días o más en los últimos 7 días.

En cuanto al hábito de consumo de tabaco, se utilizó el cuestionario Youth Risk Behavior Surveillance (Centres for Disease Control and Prevención, 2004), realizando las correspondientes adaptaciones al contexto de adolescentes españoles. De dicho cuestionario utilizamos los 11 ítems relacionados con el hábito de consumo de tabaco.

Finalmente, elaboramos un cuestionario con 30 ítems (4 ítems relacionados con la práctica físico-deportiva, 11 ítems relacionados con el consumo de tabaco y 15 items con cuestiones generales). Dicho cuestionario fue sometido a un pretest cognitivo sobre una muestra piloto de 99 adolescentes (41 mujeres y 51 varones) para determinar el nivel de comprensión de los diferentes ítems, el tiempo necesario para su administración y las dificultades de aplicación del mismo. Los resultados del pilotaje nos aconsejaron eliminar algunos ítems y modificar la redacción en algunos de ellos. Una vez establecidas las correcciones, se redactó el cuestionario definitivo que se auto-administró a los participantes.

De esta forma hemos confeccionado un cuestionario breve y de fácil comprensión que nos permitiera una comparación de resultados con las principales investigaciones nacionales e internacionales realizadas sobre dicha temática.

\section{Procedimiento}

La aplicación del instrumento se llevó a cabo sobre grupos reducidos (25-30 alumnos en aulas de clase). El permiso para el estudio fue solicitado a las autoridades escolares y a los directores de los centros. Además se les preguntó a los padres/ tutores y estudiantes sobre la participación en el estudio. Todos los sujetos participaron voluntariamente en el estudio. El cuestionario final se diseñó para ser autoadministrado, siendo el propio adolescente quien cumplimentaba los ítems planteados. El proceso se realizó bajo la supervisión de un investigador colaborador que estaba presente en todo momento para explicar el objetivo del cuestionario y la forma de cumplimentar el mismo. Dicho colaborador fue sometido a un proceso de entrenamiento que le capacitaba para poder solucionar posibles incidencias durante la aplicación del instrumento. 


\section{Análisis de los datos}

Para la obtención de los resultados de la presente investigación se ha abordado estadística descriptiva utilizando, en el caso de variables policotómicas, recuento numérico y porcentual en función del sexo y la edad de la muestra. En variables continuas se ha reflejado la media, mediana y desviación típica.

La relación entre variables categóricas se ha realizado siguiendo tablas de contingencia y aplicando $\chi^{2}$ de Pearson con el correspondiente análisis de residuos.

Se ha desarrollado estadística inferencial utilizando análisis de la covarianza (ANCOVA). Así mismo, para determinar el grado de influencia diferencial de las variables independientes constituyentes de la presente investigación, se ha desarrollado un modelo de regresión logística binaria, estableciendo como centro de referencia de puntuación "dumi" en los niveles de actividad física habitual globales y de las diferentes subescalas los puntos de corte centrales establecidos mediante percentiles.

\section{Resultados}

En relación al consumo habitual de tabaco observamos como casi un $30 \%$ de los adolescentes fuma de forma habitual, siendo las mujeres las que presentan porcentajes significativamente superiores a los hombres (tabla 2). Comprobamos que el test de independencia aplicado muestra una asociación significativa de proporción de mujeres que manifiestan un consumo habitual de tabaco respecto a los hombres. El tamaño del efecto de dicha relación viene expresado por la $\mathrm{V}$ de Cramer con un valor de $0,23(p<0,021)$.

Tabla 2. Consumo habitual de tabaco según el sexo

\begin{tabular}{|c|c|c|c|c|c|}
\hline & & & \multicolumn{2}{|c|}{ Fumador } & \multirow[b]{2}{*}{ Total } \\
\hline & & & $\mathrm{Si}$ & No & \\
\hline \multirow[t]{6}{*}{ Sexo } & Hombre & Recuento & 107 & 305 & 412 \\
\hline & & $\%$ del total & $12,7 \%$ & $36,1 \%$ & $48,8 \%$ \\
\hline & & Residuos corregidos & $-2,3$ & 2,3 & \\
\hline & Mujer & Recuento & 144 & 289 & 433 \\
\hline & & $\%$ del total & $17,0 \%$ & $34,2 \%$ & $51,2 \%$ \\
\hline & & Residuos corregidos & 2,3 & $-2,3$ & \\
\hline \multirow[t]{2}{*}{ Total } & & Recuento & 251 & 594 & 845 \\
\hline & & $\%$ del total & $29,7 \%$ & $70,3 \%$ & $100,0 \%$ \\
\hline
\end{tabular}

$\chi^{2}=5,36 p=0,021$

La proporción de fumadores habituales aumenta significativamente $\left(\chi^{2}=33,76 p<0,0005\right)$ en las diferentes franjas de edad analizadas en nuestra encuesta. Así, considerando en su conjunto al alumnado de ambos sexos, a los 14 años el 8\% de los escolares fuma habitualmente, mientras que a la edad de 17 años se incrementa hasta un 41,7\% (Figura 1). En la Figura se aprecia un fuerte incremento en el consumo habitual de tabaco de los 15 a los 16 años, pasando del 14,7\% a un 35,5\% respectivamente. El tamaño del efecto de dicha relación viene expresado por la V de Cramer con un valor de 0,21 ( $p<0,0005)$.

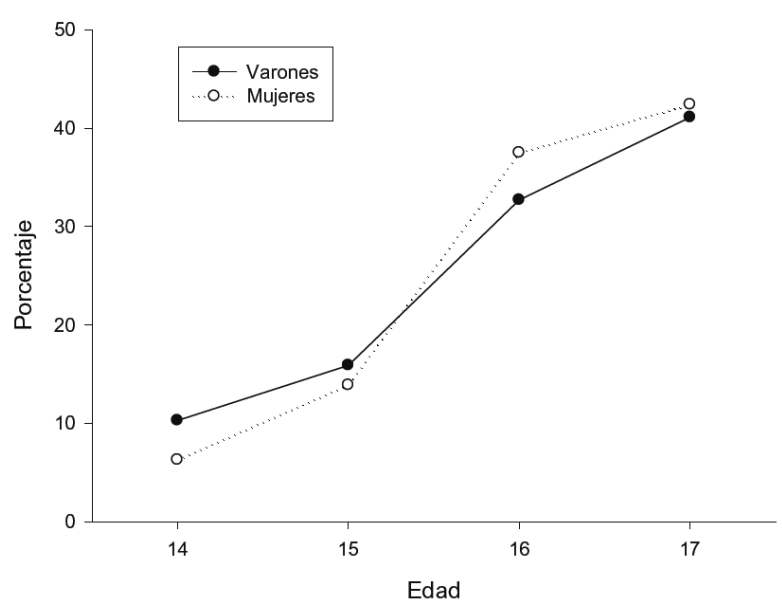

$\chi^{2}=33,76 ; p<0,0005$

Figura 1. Consumo habitual según el sexo y la edad

En la tabla 3 observamos que, de la totalidad de la muestra (845 sujetos), un $44,5 \%$ se consideran regularmente activos y un $23,9 \%$ irregularmente activo, frente al 31,6\% que es inactivo, siendo los varones los que presentan porcentajes claramente superiores, quedando reflejado en el test de independencia $\chi^{2}$ de Pearson con análisis de residuos aplicado, observando una clara asociación positiva por parte de los varones hacia la práctica deportiva superiores a las mujeres.

Tabla 3. Práctica físico-deportiva según el sexo

\begin{tabular}{|c|c|c|c|c|c|}
\hline & & & \multicolumn{2}{|c|}{ Sexo } & \multirow[b]{2}{*}{ Total } \\
\hline & & & Hombre & Mujer & \\
\hline \multirow[t]{9}{*}{ IPAQ } & \multirow[t]{3}{*}{ INACTIVO } & Recuento & 72 & 195 & 267 \\
\hline & & $\%$ del total & $8,5 \%$ & $23,1 \%$ & $31,6 \%$ \\
\hline & & $\begin{array}{l}\text { Residuos } \\
\text { corregidos }\end{array}$ & $-8,6$ & 8,6 & \\
\hline & \multirow{3}{*}{$\begin{array}{l}\text { IRREGULARMENTE } \\
\text { ACTIVO }\end{array}$} & Recuento & 103 & 99 & 202 \\
\hline & & $\%$ del total & $12,2 \%$ & $11,7 \%$ & $23,9 \%$ \\
\hline & & $\begin{array}{l}\text { Residuos } \\
\text { corregidos }\end{array}$ & ,7 &,- 7 & \\
\hline & \multirow{3}{*}{$\begin{array}{l}\text { REGULARMENTE } \\
\text { ACTIVO }\end{array}$} & Recuento & 237 & 139 & 376 \\
\hline & & $\%$ del total & $28,0 \%$ & $16,4 \%$ & $44,5 \%$ \\
\hline & & $\begin{array}{l}\text { Residuos } \\
\text { corregidos }\end{array}$ & 7,4 & $-7,4$ & \\
\hline \multirow[t]{2}{*}{ Total } & & Recuento & 412 & 433 & 845 \\
\hline & & $\%$ del total & $48,8 \%$ & $51,2 \%$ & $100,0 \%$ \\
\hline
\end{tabular}

$\chi^{2}=81,951 ; p<0,0005$

Las tablas de contingencias y la prueba de $\chi^{2}$ con análisis de residuos que relacionan el hábito de práctica físico-deportiva con el consumo de tabaco establece una asociación positiva y significativa de los varones que afirman realizar práctica físico-deportiva regular con el rechazo del hábito de fumar $\left(\chi^{2}=7,57 p=0,006\right)$. Por el contrario, en las mujeres no se aprecia esta relación entre ambas variables (tabla 4). El tamaño del efecto de dicha relación viene expresado por la $\mathrm{V}$ de Cramer con un valor de 0,31 ( $p=0,0006)$. 
Tabla 4. Pruebas de asociación (Chi-cuadrado de Pearson completada con análisis de residuos) que relacionan la práctica regular de actividad físico-deportiva y el hábito de consumo de tabaco

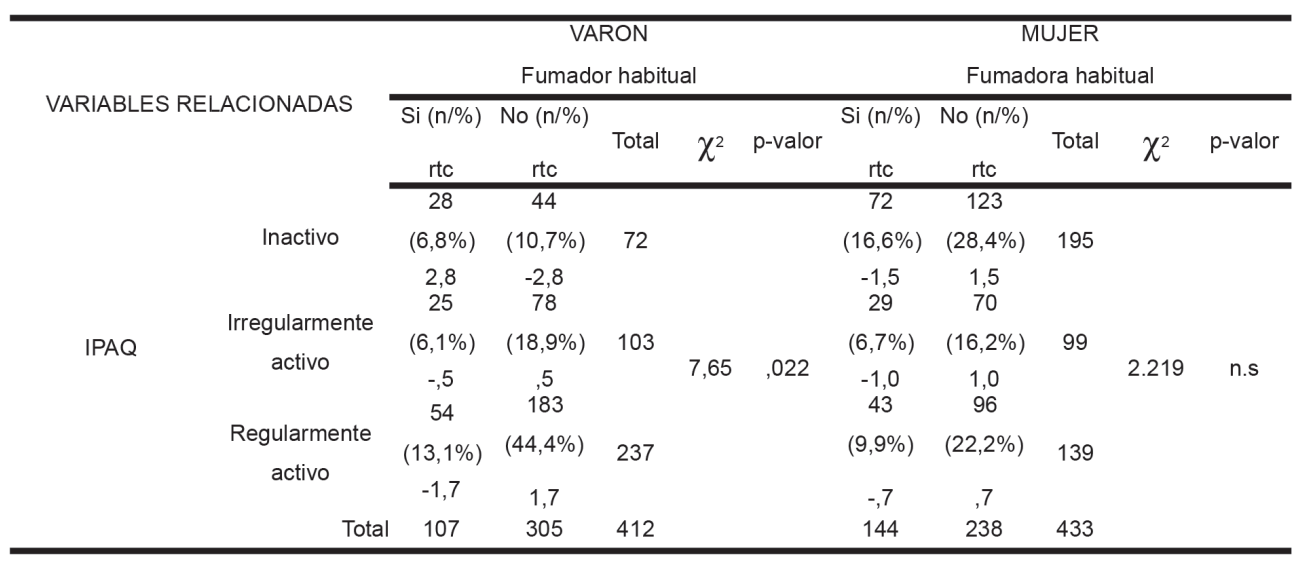

$\mathrm{N}=$ frecuencia de sujetos $\%=$ porcentaje

Rtc=residuos tipificados corregidos

2=Chi-cuadrado de Pearson

Tabla 5. Relación de la práctica regular de actividad físico deportiva con el hábito de consumo de tabaco

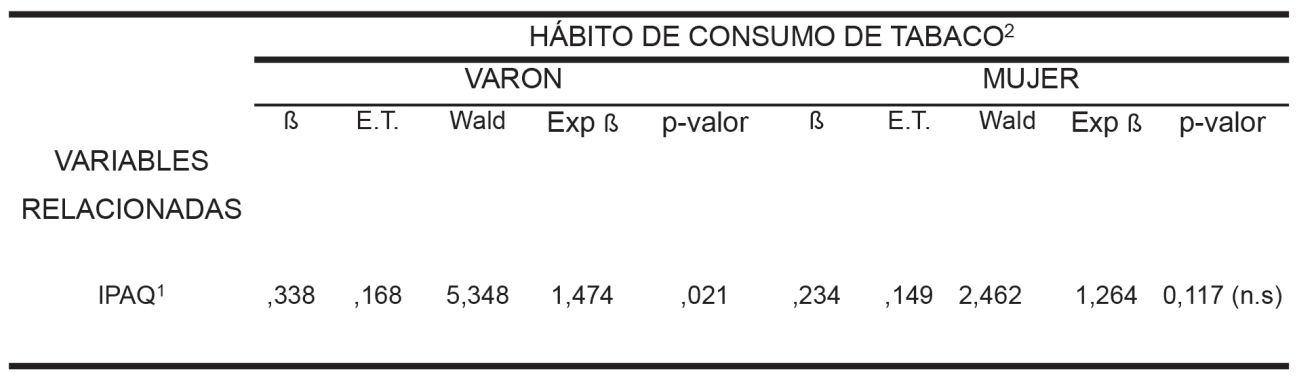

1) Practica regular de actividad fisico-deportiva: Regularmente activo $=1$; inactivo $=0$

2) Consumo de tabaco: consume tabaco de forma habitual $=0$; No consume tabaco $=1$

Esta tendencia exploratoria diferenciada en la relación de las variables se confirma en los resultados de las pruebas de regresión logística binaria aplicadas, donde los valores de B $(0,388)$ y Exp $\beta(1,474)$ con una matriz de correlación de $-0,949$ nos confirma que aquellos varones que tienden a la práctica regular de actividad físico deportiva se orientan hacia el rechazo del consumo de tabaco siendo esta relación significativa $(p=0,021)$. Por el contrario, en las mujeres esta relación no es significativa (tabla 5 ).

\section{Discusión}

Como aspecto relevante destacado en el presente trabajo señalamos la diferencia que la práctica físico-deportiva genera en el hábito de consumo de tabaco entre varones y mujeres. Los resultados de nuestra investigación constatan que la práctica regular de actividad físico-deportiva se relaciona positivamente con una menor frecuencia de consumo habitual de tabaco entre los adolescentes encuestados. Recientemente, Wilson et al. (2005) realizaron un estudio en una muestra de 10635 adolescentes de $6^{\circ}$ a $12^{\circ}$ pertenecientes al estado de Virginia (U.S.A.). El objetivo del estudio consistió en analizar la relación existente entre el consumo de tabaco, frutas, vegetales, productos lácteos y la frecuencia de ejercicio físico, encontrando que la realización de ejercicio físico ( $>3$ veces/semana) se asoció a un menor consumo de tabaco, tanto en la "Middle school-6 $6^{\circ}$ a $8^{\circ}$ " como en la "high school-9 $9^{\circ}$ a $12^{\circ}$ ". Del mismo modo, Aleixandre et al. (2005) realizaron un estudio en 1378 adolescentes de 13 a 19 años y pertenecientes a la ciudad de Mallorca, con la intención de conocer la relación existente entre los niveles de actividad y el consumo de drogas en los adolescentes, destacando que aquellos adolescentes que manifiestan realizar actividades deportivas obtienen consumos un $59 \%$ inferiores a quienes nos participan, así como unos valores de 20 veces inferiores de cantidad de cigarrillos consumidos a la semana.

En este sentido, Halperin et al. (2010) en un estudio sobre 10000 universitarios americanos señalan que cualquier nivel de consumo de tabaco fue asociado a un menor nivel de ejercicio físico. Los universitarios que suelen realizar 3 o más veces ejercicio a la semana suelen consumir menos tabaco que aquellos que lo realizan menos de 3 veces por semana.

No obstante, los datos revelan que la relación entre ambas variables difiere significativamente entre varones y mujeres. 
Consideramos que, dentro de la práctica de actividad físicodeportiva, hay que tener en cuenta diversos factores que determinan dichas variaciones. En este sentido, pensamos que las diferencias de las motivaciones y los variados contextos de práctica entre en ambos sexos poseen una gran relevancia.

En general, en cualquier contexto social de práctica físicodeportiva no es habitual la presencia de personas que consuman tabaco. Así mismo, la realización de ejercicio físico o deporte es incompatible con el acto de fumar. Diversas investigaciones han constatado los efectos perjudiciales que el consumo de tabaco posee sobre el rendimiento físico-deportivo (Bernaards, Twisk, Van-Mechelen, Snel y Kemper, 2003; Louis, 2001; Melnick, Millar, Sabo, Farell y Barnes, 2001; Nerín et al., 2004). Si tenemos en cuenta que los varones, a diferencia de las mujeres, suelen realizar práctica deportiva bajo intereses centrados en la competición, el rendimiento y el éxito deportivo, la exaltación del ego, la aprobación social o la mejora de la condición física, podemos explicarnos dicha tendencia al rechazo del consumo de tabaco (Moreno et al., 2009). Así mismo, es muy habitual que los técnicos deportivos y entrenadores inculquen en sus pupilos los perjuicios que el tabaco genera para la práctica deportiva, hecho que adquiere una especial significación en el caso de los educadores deportivos y en el ámbito de las clases de Educación Física. Dada la relevancia que estas figuras poseen en los deportistas iniciados, es fácil deducir que se produzca un efecto disuasorio en la conducta del sujeto hacia la adquisición del hábito de fumar, que puede llegar a ser más fuerte que todos los elementos personales, sociales o familiares que predisponen a su consumo. Por otro lado, es importante señalar que, en este contexto de práctica deportiva participan sujetos que comparten las mismas motivaciones e intereses, circunstancia que favorece el establecimiento de lazos de amistad que refuerzan las conductas de los adolescentes. Prueba de ello, nuestros datos señalan que, tanto en varones como en mujeres, la presencia de un entorno de amigos no fumadores se relaciona positivamente con el rechazo del hábito de consumo de tabaco. En la misma línea, Leatherdale et al. (2005) en un estudio de 22091 estudiantes de Ontario (Canadá) encuentran que existe una relación directa entre tener amigos que habitualmente consumen tabaco y ser fumador. En este sentido, Robison et al. (2006) en un estudio de 4461 estudiantes de Menphis (USA) obtienen que cerca de un tercio de los adolescentes que fuman señalan que sus amigos son fumadores. Del mismo modo, Nichols et al. (2006) en un estudio en 858 mujeres adolescentes de New York (USA) encuentran que tener amigos que fuman hace que se inicien antes en el consumo de tabaco. Igualmente, García et al. (2006) en un estudio de 529 adolescentes de 12 y 14 años encuentran que la experimentación con el tabaco se relaciona con la presencia de amigos fumadores, siendo el principal factor de riesgo para el consumo de tabaco de los adolescentes.

En cuanto a las mujeres, la mayoría de investigaciones apuntan que los motivos de práctica físico-deportiva difieren en gran medida de los varones, estando orientados preferentemente hacia la diversión, el establecimiento de relaciones afectivosociales, el cuidado de la estética, el control del peso corporal y el mantenimiento de una buena imagen. En este sentido, Crisp et al. (1998) concluyen que la ansiedad relacionada con el control del cuerpo, el miedo a sentirse demasiado gordas y a perder el control sobre la ingesta, eran factores que condicionaban el mantenimiento del consumo de tabaco entre las mujeres adolescentes. Así mismo, 0'Loughlin et al. (1998) en un estudio sobre 1824 adolescentes de entre 9 y 12 años pertenecientes a la ciudad de Montreal (Canadá), encontraron que las adolescentes utilizaban el tabaco como elemento para controlar su peso. En este sentido, no encontraron relación entre el control del peso y el inicio en el consumo de tabaco, mientras que sí lo relacionaron con el mantenimiento del hábito de consumo, de tal forma que las adolescentes que presentan sobrepeso fumaban tres veces más que aquellas que no presentaban dicho problema estético. Por su parte, Tomeo et al. (1999) en un estudio sobre 16862 adolescentes norteamericanos de 9 a 14 años, encontraron que los sujetos encuestados afirmaron que uno de los motivos para iniciarse en el consumo de tabaco era el control del peso corporal. En este sentido, Quiles et al. (2011) en un estudio sobre 2142 adolescentes de Educación Secundaria Obligatoria encontraron una relación positiva entre el consumo de tabaco y la pérdida de peso en las adolescentes que presentan riesgo de desarrollar un trastorno de conducta alimentaria. Las adolescentes que presentan un mayor riesgo de trastorno alimentario consumen más cantidad de tabaco que aquellas que presentan un riesgo menor, estando claramente relacionado el consumo de tabaco con la intención de perder peso.

Estamos asistiendo a una clara incorporación de la mujer al hábito de consumo de tabaco, provocado por el estrés y por la relación existente entre fumar y las actitudes de preocupación por la imagen corporal (Chen et al., 2005; Froom, Relamed y Benbassat, 1998; Strauss y Mir, 2001). En este sentido, Chatkin y Chatkin (2007) señalan el efecto directo de la nicotina sobre el metabolismo del tejido graso causando una mayor oxidación del tejido graso, lo que nos ayuda a explicar el hecho de que el índice de masa corporal sea menor en los fumadores. Por otro lado, De la Rosa y Otero (2004) y Nerín (2005) señalan que la progresiva incorporación de la mujer al ámbito laboral está influyendo de forma directa en el aumento de este hábito nocivo.

Si tenemos en cuenta que el consumo de tabaco en la mujer tiene una relación directa con el cuidado de la imagen corporal y el control del sobrepeso, es fácil que la práctica de ejercicio físico se desarrolle asociada al consumo de tabaco, sobre todo, en contextos de práctica físico-deportiva donde la competición y el rendimiento no son relevantes. Este hecho nos puede explicar las diferencias establecidas entre los varones y mujeres fumadores en relación con la cantidad de tabaco consumida a la semana. Las adolescentes que no realizan actividad físicodeportiva presentan valores de consumo significativamente más elevados que aquellas que practican de forma regular. Pensamos que la actividad física puede generar un efecto coadyuvante sobre el control de la imagen corporal y el mantenimiento del peso corporal, circunstancia que reduce las cantidades de tabaco consumidas. Por el contrario, aquellas adolescentes que son sedentarias necesitan consumir más tabaco para conseguir controlar su peso corporal. En el caso de los varones fumadores, esta relación no es significativa y, por tanto, consideramos que está relacionada con otros factores predisponentes. Así mismo, el descenso de la práctica deportiva y de los niveles de actividad física habitual con la edad pensamos que tienen una relación directa con el aumento de adolescentes fumadores. 
Estando relacionada la práctica físico-deportiva con la reducción en la proporción del número de fumadores en la etapa adolescente, consideramos que se deben abordar políticas de promoción deportiva que favorezcan la participación de los mismos. Sobre todo, se deben redoblar los esfuerzos en el caso de las mujeres, ya que el abandono de la práctica deportiva y el aumento en consumo habitual de tabaco en ellas son alarmantes. Así mismo, es fundamental ofrecer modelos de práctica deportiva adaptada a las motivaciones diferenciales establecidas entre varones y mujeres. De este modo, consideramos fundamental promocionar las actividades físico-deportivas durante el tiempo de ocio, siendo para ello necesario establecer una intervención multidisciplinar entre los principales responsables del deporte juvenil. Sería preciso establecer proyectos coordinados entre las administraciones regionales, las instituciones educativas y las administraciones locales encaminados a aumentar las franjas horarias y los programas de actividades dirigidos hacia los adolescentes.

Como limitación de nuestra investigación hemos de señalar que no se han analizado los motivos diferenciales entre varones y mujeres a la hora de realizar práctica físico-deportiva. Estas diferencias de motivaciones han sido constatadas en numerosas investigaciones que han sido referenciadas en el presente estudio y que pueden constituir una posible explicación de las diferencias por sexo en relación al hábito de consumo de tabaco y la práctica físico-deportiva en adolescentes escolarizados.

\section{Reconocimientos}

A todo el profesorado de los centros de Educación Secundaria donde se realizaron las encuestas, en especial a los departamentos de Educación Física que con su ayuda nos han facilitado la posibilidad de realizar el estudio.

A la Consejería de Educación, Formación y Empleo que nos facilitó los permisos para realizar el estudio en los centros seleccionados.

\section{Conflicto de intereses}

Los autores no tienen conflictos de intereses que declarar.

\section{Referencias}

Aleixandre, N. L., Perello, M. J. y Palmer, A. L. (2005). Activity levels and drug use in a simple of Spanish adolescents. Addictive Behaviors, 30, 1597-1602. doi:10.1016/j.addbeh.2005.02.009.

Audrian-McGorven, J., Rodríguez, D. y Moss, H. (2003). Smoking progression and physical Activity. Cancer Epidemiology Biomarkers Prevention, 12, 1121-1129.

Audrian-Mcgovern, J., Rodriguez, D., Wileyto, P., Schmitz, K. H. y Shields, P. G. (2006). Effect of team sport participation on genetic predisposition to adolescent smoking progression. Archives of General Psychiatry, 63, 433-441. doi:10.1001/archpsyc.63.4.433.

Bello, S. (2011). Tratamiento del tabaquismo. Revista Chilena de Cardiología, 30, 230-239.
Bergamaschi, A., Morri, M., Resi, D., Zanetti, F. y Stampi S. (2002). Tobacco consumption and sports participation: a survey among university students in northern Italy. Annali de Ingieni, 14, 435-442.

Bernaards, C. M., Twisk, J. W., Van Mechelen, W., Snel, J. y Kemper, H. C. (2003). A longitudinal study on smoking in relationship to fitness and heart rate response. Medicine \& Science in Sports and Exercise, 35, 793-800. doi:10.1249/01.MSS.0000064955.31005.E0.

Bungum, T., Dowda, M., Weston, A., Trost, S. G. y Pate, R. (2000). Correlates of physical activity in male and female youth. Pediatric Exercise Science, 12, 71-79.

Castillo I. y Balaguer, I. (2004). Dimensiones de los motivos de práctica deportiva de los adolescentes valencianos escolarizados. Apunts, 63, 22-29.

Cechini, J. A., Méndez, A. y Muñiz, J. (2003). Tendencias o direcciones del deporte contemporáneo en función de los motivos de práctica. Apunts, 72, 6-13.

Centers for Disease Control and Prevention (2004). Youth Risk Behavior Surveillance. MMWR Surveillance Summaries, 53, 1-16. Disponible en: http://www.cdc.gov/mmwr/PDF/rr/rr5312.pdf.

Chatkin, R., Chatkin, J. M. (2007). Smoking and changes in body weight: can physiopathology and genetics explain this association?. Jornal Brasileiro de Pneumologia, 33, 712-719.

Chen, H., Vlahos, R., Bozinovski, S., Jones, J., Anderson, G. P. y Morris, M. J. (2005). Effect of short-term cigarette smoke exposure on body weight, appetite and brain neuropeptide $Y$ in mice. Neuropsychopharmacology, 30, 713-719. doi:10.1038/ sj.npp. 1300597.

Colegio Oficial de Psicólogos-International Tests Comission (2000). Directrices internacionales para el uso de los tests. Madrid, España: Autor.

Colmen, T. L., Barrett, E., Clausen, J., Holemn, J. y Bjermer, L. (2002). Physical exercise, sports, and lung function in smoking versus nonsmoking adolescents. European Respiratory Journal, 19, 8-15.

Craig, C. L., Marshal, A. L., Sjostrom, M., Bauman, A. E., Booth, M. L. y Ainsworth, B. E. (2003). International physical activity questionnaire: 12-country reliability and validity. Medicine \& Science in Sports and Exercise, 35, 1381-95. doi:10.1249/01.MSS.0000078924.61453.FB

Crisp, A. H., Stavrakaki, C., Halek, C., Williams, E., Segwick, P. y Kiosiss, I. (1998). Smoking and pursuit of thinness in schoolgirls in London and Ottawa. Postgraduate Medical Journal, 74, 473-479.

Daniel, J., Cropley, M., Ussher, M. y West, R.. (2004). Acute effects of a short bout of moderate versus light intensity exercise versus inactivity on tobacco withdrawal symptoms in sedentary smokers. Psychopharmacology, 174, 320-326. doi:10.1007/s00213-003-1762-x.

De la Rosa, L. y Otero, M. (2004). Tabaquismo en la mujer: consideraciones especiales. Trastornos adictivos, 6, 113-124.

Ford, C. A., Nonnemaker, J. M. y Wirth, K. E. (2008). The influence of adolescent body mass index, physical activity, and tobacco use on blood pressure and cholesterol in young adulthood. Journal of Adolescent Health, 43, 576-583. doi:10.1016/j. jadohealth.2008.06.010.

Froom, P., Melamed, S. y Benbassat, J. (1998). Smoking cessation and weight gain. The Journal of Family Practice, 46, 460-464. 
Garcia, P., Carrillo, A., Fernández, A. y Sánchez, J. M. (2006). Factores de riesgo en la experimentación y el consumo de tabaco en estudiantes de 12 a 14 años. Actitudes ante el tabaco en los grupos de presión. Atenención Primaria, 37, 392-399.

Gómez, L. F., Duperly, J., Lucumi, D. I., Gámez, R. y Venegas, A. S. (2005). Nivel de actividad fisica global em La población adulta de bogotá (Colombia). Prevalencia y factores asociados. Gaceta Sanitaria, 19, 206-213.

Halperin, A. C., Smith, S. S., Heligenstein, E., Brown, D. y Fleming, M. F. (2010). Cigarette smoking and associated health risk among students at five universities. Nicotine \& Tobacco Research, 12, 96-104. doi: 10.1093/ntr/ntp182.

Iannotti, R. J., Kogan, M. D., Janssen, I. y Boyce, W. F. (2009). Patterns of adolescent physical activity, screen-based media use, and positive and negative health indicators in the USE and Canada. Journal of Adolescent Health, 44, 493-499. doi: 10.1016/j. jadohealth.2008.10.142.

Larson, N. I., Story, M., Perry, C. L., Neumark-Sztainer, D. y Hannan, P. J. (2007). Are diet and physical activity patterns related to cigarette smoking in adolescents? Findings from project EAT. Preventing Chronic Disease, 4, 1-12.

Leatherdale, S., McDonald, P., Cameron, R. y Brown, K. (2005). A multinivel analysis examining the relationship between social influences for smoking and smoking onset. American Journal of Health Behavior, 29, 520-530. doi: 10.5993/ajhb.29.6.7.

Lekuona, I., Salcedo, A., Morillas, M. y Umaran, J. (2009). Tabaco y enfermedad arterial no coronaria. Intervenciones para el abandono del hábito tabáquico. Revista Española de Cardiología, 9 (Supl.D), 39-48.

Levin, S., Lowry, R., Brown, D. V. y Dietz, W. H. (2003). Physical activity and body mass index among US adolescents: youth risk behavior survey, 1999. Archive of Pediatrics \& Adolescent Medicine, 157, 816820. doi: 10.1001/archpedi.157.8.816.

Louie, D. (2001). The effects of cigarette smoking on cardiopulmonary function and exercise tolerance in teenagers. Canadian Respiratory Journal, 8, 289-291.

Madan, A. K., Barden, C. B., Beech, B., Fay, K., Sintich, M. y Beech, D. J. (2005). Multivariate analysis of factors associated with smoking cessation in women. Journal of the Louisiana State Medical Society, 157, 112-115.

Melnick, M. J., Miller, K. E., Sabo, D. F., Farrell, M. P. y Barnes, G. M. (2001). Tobacco use among high school athletes and nonathletes: results of the 1997 yoth risk behavior survey. Adolescence, 36, 727-747.

Moore, M. y Werch, Ch. E. (2005). Sport and physical activity participation and substance use among adolescents. Journal of Adolescent Health, 36, 486-493. doi:10.1016/j.jadohealth.2004.02.031.

Moreno, J. A., Moreno, R. y Cervelló, E. (2009). Relación del autoconcepto físico con las conductas de consumo de alcohol y tabaco en adolescentes. Adicciones, 21, 147-154.

Muñiz, J. (1996). Directrices para la traducción y adaptación de los tests. Papeles del psicólogo, 66, 63-70.

Nerín, I. (2005). El tabaquismo en la mujer: una atracción fatal. Archivos de Bronconeumología, 41, 360-362.
Nerín, I., Crucelaegui, A., Novella, P., Ramón, P., Sobradiel, N. y Gerico R. (2004). Encuesta sobre tabaquismo en estudiantes universitarios en relación con la práctica de ejercicio físico. Archivos de Bronconeumología, 40, 5-9.

Nichols, T. R., Birnbaum, A. S., Birnel, S. y Botvin, G. J. (2006). Perceived smoking environment and smoking initiation among multi-ethnic urban girls. Journal of Adolescent Health, 38, 369-75. doi:10.1016/j. jadohealth.2005.04.016.

Nistal, P., Prieto, J. A., Del Valle, M. y González, V. (2003). Relación de la actividad física con el consumo de tabaco en adolescentes. Archivos de Medicina del Deporte, 20,397-403.

O'Loughlin, J., Paradis, G., Renaud, L. y Sanchez, L. (1998). One-year predictors of smoking initiation and continued smoking among elementary schoolchildren in multiethnic, low-income, inner-city neighbourhoods. Tobacco Control, 7, 268-275.

Organización Mundial de la Salud (1997). Declaración de Yacarta. Organización Mundial de la Salud.

Pate, R. R., Saunders, R., Dishman, R. K., Addy, C., Dowda, M. y Ward, D. S. (2007). Long-term effects of a physical activity intervention in high school girls. American Journal of Preventive Medicine, 33, 276-280. doi:10.1016/j.amepre.2007.06.005.

Quiles, Y., Balaguer, I., Pamies, L., Quiles, M. J., Marzo, J. C. y Rodríguez, J. (2011). Eating habits, physical activity, consumption of substances and eating disordes in adolescents. The Spanish Journal of Psychology, 14, 712-723. doi: 10.5209/rev_sjop.2001.v14.n2.19.

Robinson, L. A., Dalton, W. T. y Nicholson, L. M. (2006). Changes in adolescent's sources of cigarrettes. Journal of Adolescent Health, 39, 861-867. doi:10.1016/j.jadohealth.2006.06.004.

Sanchez, A., Norman, G. J., Sallis, J. F., Calfas, K. J., Cella, J. y Patrick, K. (2007). Patterns and correlates of physical activity and nutrition behaviors in adolescents. American Journal of Preventive Medicine, 32, 124-130. doi:10.1016/j.amepre.2006.10.012.

Strauss, R. S. y Mir, H. M. (2001). Smoking and weight loss attempts in overweight and normal-weight adolescents. International Journal of Obesity, 25, 1381-1385.

Suelves, J. M., Romero, R. y Sánchez, M. (2000). Prevención del abuso de drogas en la escuela secundaria tras la reforma educativa. Implantación de diversos programas en la ciudad de Barcelona. Gaceta Sanitaria, 14, 131-138.

Telama, R., Yang, X., Viikari, J., Välimäki, I., Wanne, O. y Raitakari, 0. (2005). Physical activity from childhood to adulthood: a 21-year tracking study. American Journal of Preventive Medicine, 28, 267273. doi:10.1016/j.amepre.2004.12.003.

Tomeo, C. A., Field, A. E., Berkey, C. S., Colditz, G. A. y Frazier, A. L. (1999). Weight concerns, weight control behaviors, and smoking initiation. Pediatrics, 104, 918-924.

Wilson, D. B., Smith, B. N., Speizer, I. S., Bean, M. K., Mitchell, K. S., Uguy, L. S. y Fries, E. A. (2005). Differences in food intake and exercise by smoking status in adolescents. Preventive Medicine, 40, 872-879. doi: 10.1016/j.ypmed.2004.10.005.

World Health Organization. International Classification of Diseases, 10th Edn. Geneva, World Health Organization, 1992. Disponible en: http://www.who.int/classifications/icd/en/GRNBOOK.pdf. 